\title{
A GFP Positive Glioblastoma Cell Line NS1 - A New Tool for Experimental Studies
}

\author{
Henrietta Nittby ${ }^{1,2 *}$, Karolina Förnvik ${ }^{1 \#}$, Jonatan Ahlstedt ${ }^{1 \#}$, Crister Ceberg ${ }^{3}$, Peter Ericsson ${ }^{1}$, Bertil R Persson ${ }^{3}$, Gunnar Skagerberg $^{1}$, Bengt Widegren $^{1}$, \\ Zhongtian Xue ${ }^{1}$ and Leif G Salford ${ }^{1,2}$ \\ ${ }^{1}$ The Rausing Laboratory, BMC D10, Lund University, SE-223 62 Lund, Sweden \\ ${ }^{2}$ Department of Neurosurgery, Skåne University Hospital in Lund, SE 221-85 Lund, Sweden \\ ${ }^{3}$ Department of Medical Radiation Physics, Lund University, SE-221 85 Lund, Sweden
}

*Corresponding author: Henrietta Nittby, Department of Neurosurgery, Skåne University Hospital in Lund, SE 221-85 Lund, Sweden, Tel: +4646173922; E-mail: henrietta.nittby@med.lu.se

Rec. Date: Sep 05, 2015; Acc. Date: Sep 21, 2015; Pub. Date: Sep 25, 2015

Copyright: @ 2015 Nittby et al. This is an open-access article distributed under the terms of the Creative Commons Attribution License, which permits unrestricted use, distribution, and reproduction in any medium, provided the original author and source are credited.

\#Contributed equally

\begin{abstract}
One of the key problems when treating patients with malignant gliomas is that despite being able to remove the major bulk of the tumour, tumour cells may have already spread to distant sites of the brain. The aim of the present study is to develop a GFP positive tumour cell line (named NS1) where these cells could be identified for future treatment studies in fully immunocompetent animals.

Five homozygous GFP positive Fischer 344 rats were treated with ENU during pregnancy. Of the offspring, 19 rats developed CNS tumours. Of these, we chose those with tumours growing intracerebrally for further culturing. The tumour cells were transplanted to new recipients. Out of these, we have defined a cell line (NS1), which originated in pons, and reliably generates tumours when transplanted s.c. and i.c.

Our new GFP positive cell line was transplanted into GFP negative Fischer 344 rats. The tumours were positive for GFP. With htx-eosin staining, cell rich tumours could be identified both i.c. and s.c. Interestingly, GFAP expression was clear upon i.c. transplantation, but not in the s.c. tumours. The tumour cells had a strong RNA expression for wt IDH1, wt p53, IDO1 and EGFR and to a somewhat smaller extent also to PDL1. Upon irradiation with $8 \mathrm{~Gy}$, IDO1 expression was decreased.

With a new GFP positive tumour cell line we have a new tool for investigations of the migrating tumour cells in experimental model. With this, our hope is that treatment protocols will more easily evaluate not only the effect of survival (which is often a limited number of days in animal models) but also whether satellite cells are targeted or not. Of special interest is that the tumour cells can be used in fully immunocompetent animals, meaning that the setting is more similar to a clinical picture.
\end{abstract}

Keywords: Glioblastoma; Tumour model; GFP; Cell migration

\section{Introduction}

Gliomas are malignant tumors of the Central Nervous System (CNS) derived from glial lineage. They constitute more than $50 \%$ of all primary human intracranial tumors. Despite substantial technical improvements of conventional neurosurgery, chemotherapy and radiation therapy, the survival for glioma patients is still very low [1].

Preclinical evaluation of novel treatments for glioma requires accuracy and reproducibility in tumor models. Syngeneic intracranial brain tumor models like the F98 and RG2 lines are excellent tools in this aspect, and are widely used because of their high reproducibility and non-immunogenicity. Previously, the 9L gliosarcoma model has been frequently used, but it has since been shown to be strongly immunogenic, and the value of this model has been put into question [2].

The infiltrative growth pattern of glioblastomas, with migrating malignant cells and diffuse growth peripherally to the main tumor mass makes radical tumor resections impossible in the clinical setting. Being able to visualize the effect of treatment on satellite tumor cells may have a large impact on the clinical outcome of studied treatment protocols, and therefore it could be stated that there is need for a model where this is possible. We have therefore developed a GFP positive tumor line, to aid researchers in evaluating these aspects of glioblastoma treatments. We also mean that it is of importance to be able to use fully immunocompetent animals in the glioma model.

Verreault et al. [3] transduced U251iMG cells to generate cells expressing red fluorescent protein. However, the fluorescence was highly variable in the cells and cells with high levels of fluorescence grew much slower in vivo and in vitro. Also, cells with high fluorescence levels could not grow when transplanted into mice, but actually regressed. It was concluded that since fluorescence signal intensity could be measured in the brain of live animals, it could be used to assess tumour progression.

Bryant et al. [4] transfected F98 cells were with the firefly luciferase gene and injected them into the brains of rats. This means that all cells 
had to undergo the transfection process prior to injection and tumour did not grow in all of the rats, which actually is a problem if the model is to be used to study the effect of anti-tumour treatment. Also, in some rats tumours grew distal to the injection site and could no be followed. However, for those rats where tumours actually were growing and could be followed, an increasing luciferase signal could be followed over a period of 4 weeks, until it dropped due central necrosis.

Tumours were induced in pregnant GFP positive rats with treatment of ENU (etylnitrosurea), which has been described by Wechsler et al., with a high selectivity for the nervous system and high incidence of CNS tumours in the offspring [5]. The aforementioned study described that when ENU was administered transplacentally, 92\% of the offspring developed CNS tumours, however only when ENU was administered after the 12th day of gestation. Therefore, we treated female pregnant GFP positive rats as described below with ENU and were able to establish a new tumour cell line NS1 (Nittby-Salford-1) with capacity to grow both i.c. and s.c. We here describe both immunohistochemical aspects of the tumour, as well as PCR findings of IDH1, IDO1, EGFR, p53 and PDL1. Since radiotherapy is commonly used in the clinical setting, we also examined the effect of radiotherapy upon PCR findings of IDH1, IDO1, EGFR, p53 and PDL1.

EGFR has been shown to be overexpressed in $90 \%$ of GBM specimens, and amplification of EGFR has implications for tumour progression. Upon activation of EGFR, survival mechanisms and cellular mitogenesis are induced. Vaccination studies against EGFR are ongoing, and it has been argued that a T-cell response can be elicited against the tumour cells with these kinds of vaccines [6].

Low-grade diffuse gliomas WHO grade II (diffuse astrocytoma, oligoastrocytoma, oligodendroglioma) are characterized by frequent IDH1/2 mutations (80\%) that occur at a very early stage [7]. So called primary (de novo) glioblastomas with IDH1/2 mutations are very rare (less than 5\%). The mutation of IDH1 in a high grade glioma indicates that this is a so called secondary glioblastoma, that is a low grade astrocytoma which has progressed into a high grade astrocytoma. Furthermore, it has been shown that p 53 mutations are more common in secondary glioblastomas than in de novo glioblastomas. Therefore, the characterization of IDH1 and p53 in our new tumour model is important in order to show whether it has characteristics more of a primary than a seconardy glioma.

IDO1 (indoleamine 2,3-dioxygenase) catalyzes tryptophan metabolism and plays a key role in the T-cell dependent immune tolerance of semiallogeneic fetus, an effect mediated through tryptophan depletion. It has been shown that $85 \%$ of human gliomas express IDO1 [8]. Programmed-death-1 (PD-1, CD279), an immune checkpoint surface receptor expressed on lymphocytes, has been implicated as a mediator of immune suppression in a variety of tumors, including GBM. Binding of PD-1 to its ligands B7-H1 (PD-L1) or B7DC (PD-L2) induces apoptosis or exhaustion of activated immune cells, and blocking this in GBM models has been shown to increase survival [9]. Also, it has been shown that human gliomas express PDL1 [10].

\section{Material and Methods}

\section{Ethics statement}

All animal procedures were performed according to the practices of the Swedish Board of Animal Research and were approved by the
Animal Ethics Committee, Lund-Malmö, Sweden (Permit number: M310-10). All efforts were made to minimize suffering.

\section{Animals}

Homozygous GFP positive Fischer 344 rats as well as GFP negative Fischer 344 rats were kept at a 12-h light and 12-h dark cycle in rat hutches (2-3 in each cage) with free access to standard rodent chow and tap water. All surgery was performed under isoflurane inhalation anaesthesia. No antibiotics were used. The GFP positive animals had a promoter (ubiquitin c) that produced endogenous levels of the GFP protein at a single copy insert. Animals were sacrifice with $\mathrm{CO} 2$ inhalation when they showed signs of tumour growth such as paresis or epilepsy.

\section{Development of a GFP positive tumour cell line (named NS1)}

Pregnant GFP positive female rats, with homozygous GFP positive offspring, were treated with $60 \mathrm{mg} / \mathrm{kg}$ of ENU i.v. at estimated gestation day 15. The offspring was followed for signs of tumour growth, including paresis, epilepsy or a declined in general condition. When this was observed, the animals were euthanasised and the brain and medulla were dissected.

Once a tumour line was established the tumour cells were transplanted to new recipients. The cells were transplanted intracerebrally ( $2 \mathrm{~mm}$ from midline and $1 \mathrm{~mm}$ anterior to the coronary suture, at a depth of $5 \mathrm{~mm}$ using a stereotactic frame). For subcutaneous implantation, cells were injected s.c. in the right flank. The tumour cells were transplanted to new recipients. Out of these, we have defined a cell line (NS1), which originated in pons, and reliably generates tumours when transplanted s.c. and i.c.

\section{Cell line}

RG2 cell lines were used as controls (induced by Wechsler [5], described by Aas et al. [11]). Irradiation was performed with, a single absorbed dose of 8 Gy 137Cs-gamma rays administered to both the RG2 cell line and NS1 cell line described above. Cells were cultured in RPMI-1640 medium supplemented with $10 \%$ fetal calf serum, HEPES and $0.5 \mathrm{mM} \mathrm{Na}$-pyruvate and gentamycin.

\section{Detection of the gene expression using RT-PCR}

The gene expression of the tumor cells was studied with RT-PCR for the following genes IDH1, IDO1, EGFR, p53, and PDL1 by using HPRT1 as housekeeping gene och RG2 cells and NS1 cells passed three times subcutaneously through host animals.

RT-PCR analysis was performed using the kit Super-script one-step RT-PCR with Platinum Taq (Invitrogen) according to the instructions. The primer sequences for the different genes were designed using Gene Fisher software support (G. Giegerich, F. Meyer, C. Schleiermacher, ISMB-96). The sequences of forward primers and reverse primers for the genes analyzed, respectively, were: rPDL1-F: 5'ACGCAGGTGTCTACT-GCTGCA-3', rPDL1-R: 5' CGCTGGTCACGTTGAGAAGCT-3'; rEGFR-F: 5' CTGGAAGACCGGCGTTTGGT-3', rEGFR-R: 5'-CCATAGCTCCAGACGTCGCTT-3'; rP53-F: 5'-GTATGCTGAGTA-TCTGGACGA-3', rP53-R: 5'-AACACGAACCTCAAAGCTGT-3'; rIDH1-F: 5'-TGGTGATGTGCAGTCAGACTC-3', rIDH1-R: 5'-CAAGCTTTGCTCTGTGGGCTA-3'; rIDO1-F: 5'-GTTCTTCGCAT-ATATTTGTCTG-3', rIDO1-R: 5'-TGGCCTGCACTGCCCCCTG-3'; rHPRT-F: 5'-CAAG- 
Citation: Nittby H, Fornvik K, Ahlstedt J, Ceberg C, Ericsson P, et al. (2015) A GFP Positive Glioblastoma Cell Line NS1 - A New Tool for

Page 3 of 8

CTTGCTGGTGAAAAGGA-3', rHPRT-R: 5'-CACAAACATGATTCAAATCCCTGA-3'. The PCR conditions was set as follows: 1 denaturing cycle at $94^{\circ} \mathrm{C}$ for 2 min followed by 40 cycles at $94^{\circ} \mathrm{C}$ for $15 \mathrm{~s}, 50^{\circ} \mathrm{C}-57^{\circ} \mathrm{C}$ (according to the requirement of different genes) for $30 \mathrm{~s}$, and $72^{\circ} \mathrm{C}$ for 30 s with a final extension reaction at $72^{\circ} \mathrm{C}$ for $5 \mathrm{~min}$.

The RT-PCR products were electrophoresed on a $1 \%$ agarose gel containing Gel-Red and visualized under UV lights. The RT-PCR products as the DNA bands were cut out from the gel and purified by using MicroElute Gel Extraction kit (OMEGA bio-tek).

The concentrations of the purified RT-PCR products were measured by Bio-Photometer (Eppendorf) and the equivalent copy number of each RT-PCR product was calculated. Based on the copy number, a series of dilutions was made. They were used as the samples for establishing the standard curve that used in qRT-PCR.

\section{Quantitative real time PCR analysis}

The qRT-PCR analyses were performed using the SuperScript III Platinum Two-Step qRT-PCR Kit with SYBR Green (Invitrogen).

A total of $1 \mu \mathrm{g}$ total RNA was used in a $20 \mu \mathrm{l}$ RT reaction using a mixture of polydT and random hexamers primers. The cDNA obtained was diluted to a total volume of $80 \mu \mathrm{l}$ and stored at $-20^{\circ} \mathrm{C}$. The primers used in the qRT-PCR were the same as those in the RT-PCR.

The qRT-PCR was performed in $20 \mu \mathrm{l}$ reaction consisting of $2 \mu \mathrm{l}$ diluted cDNA or standard DNA samples, $0.3 \mathrm{M}$ of each primer, $1 \mathrm{x}$ Bovine Serum Albumin $(50 \mu \mathrm{g} / \mathrm{ml})$ and $10 \mu$ Platinum SYBR Green qRT-PCR superMix-UDG. Amplification of the target genes was carried out in a Light Cycler (Roche Molecular Biochemical) with the following thermal profile: UDG incubation at $30^{\circ} \mathrm{C}$ for $2 \mathrm{~min}$, then denaturation at $95 \circ \mathrm{C}$ for $5 \mathrm{~min}$, followed by 45 cycles at $94^{\circ} \mathrm{C}$ for $8 \mathrm{~s}$, $50-57^{\circ} \mathrm{C}$ (according to the requirement of different genes) for $10 \mathrm{~s}$, and $72^{\circ} \mathrm{C}$ for $14 \mathrm{~s}$. After amplification quantification and melting curve analysis were performed using the Light Cycler software (Roche Molecular Biochemical). The qRT-PCR samples were run in triplicate.

\section{Histopathology}

After dissection, the brains were immersion-fixed in $4 \%$ buffered formalin. The brains were serially sectioned at 40 micrometers and stored in anti-freeze. Sections of the brains were stained with Haematoxylin and Eosin and examined in the microscope. Additional sections were made of paraffin embedded brain tissue samples, and sectioned at 5 micrometers.

Other sections were incubated with a rabbit monoclonal antibody against GFAP (antibodies online) or GFP (antibodies online) at a dilution of 1:200 for two hours and subsequently with a biotinylated secondary antibody and $\mathrm{ABC}$ reagent 30 minutes each, these two later steps were carried out using a ready-to use Vectastain $\mathrm{ABC}$ kit (Vector Laboratories, CA, USA) whereafter the antigen-antibody complex was visualized using the DAKO Liquid DAB Substrate-Chromogen System (DAKO, CA, USA). The sections were thoroughly rinsed in PBS before each step of the immunohistochemical procedure.

\section{Results}

Five pregnant rats were treated with ENU, which generated an offspring of 42 animals, out of which 19 rats developed CNS tumours. Of these, we chose those with tumours growing intracerebrally for further culturing (12 rats), meaning that tumours in medulla spinalis
(TMS) were not considered for our glioma cell lines in these first experiments.

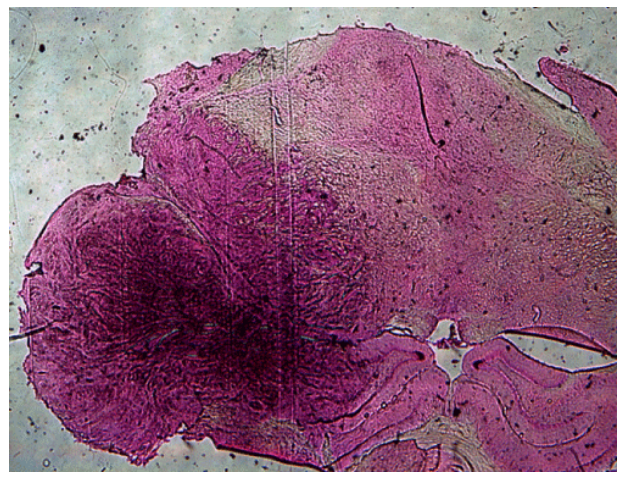

Figure 1: Htx-eosin staining of intracerebrally inplanted NS1 tumour cells.

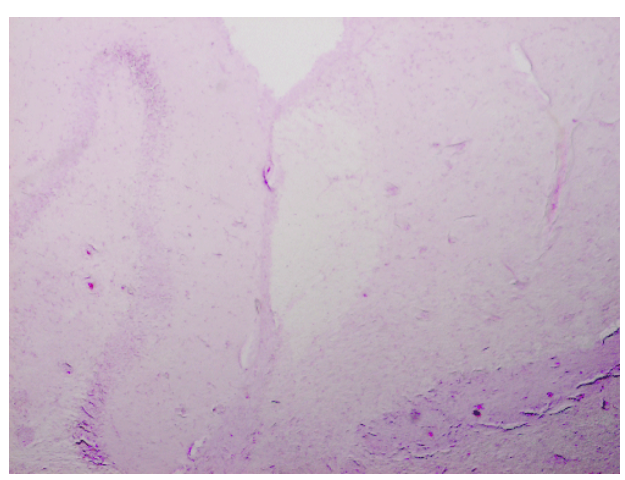

Figure 2: Htx-eosin staining of intracerebrally inplanted NS1 tumour cells.

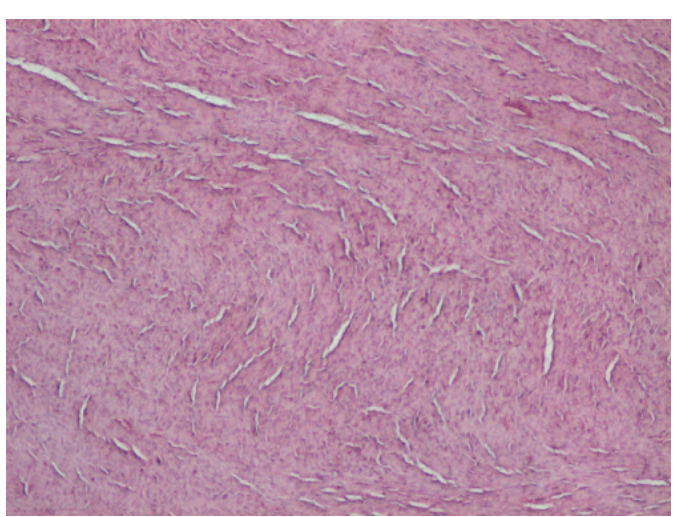

Figure 3: Htx-eosin staining of sc inplanted NS1 tumour cells. 
Citation: Nittby H, Fornvik K, Ahlstedt J, Ceberg C, Ericsson P, et al. (2015) A GFP Positive Glioblastoma Cell Line NS1 - A New Tool for

Page 4 of 8

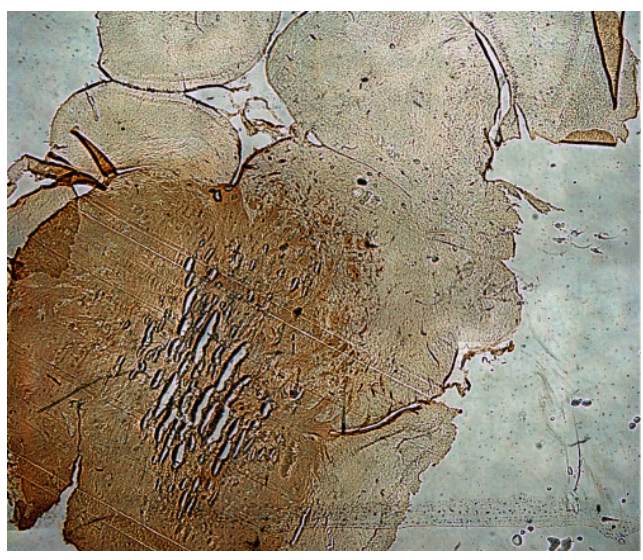

Figure 4: Anti-GFP staining of intracerebrally inplanted NS1 tumour cells.

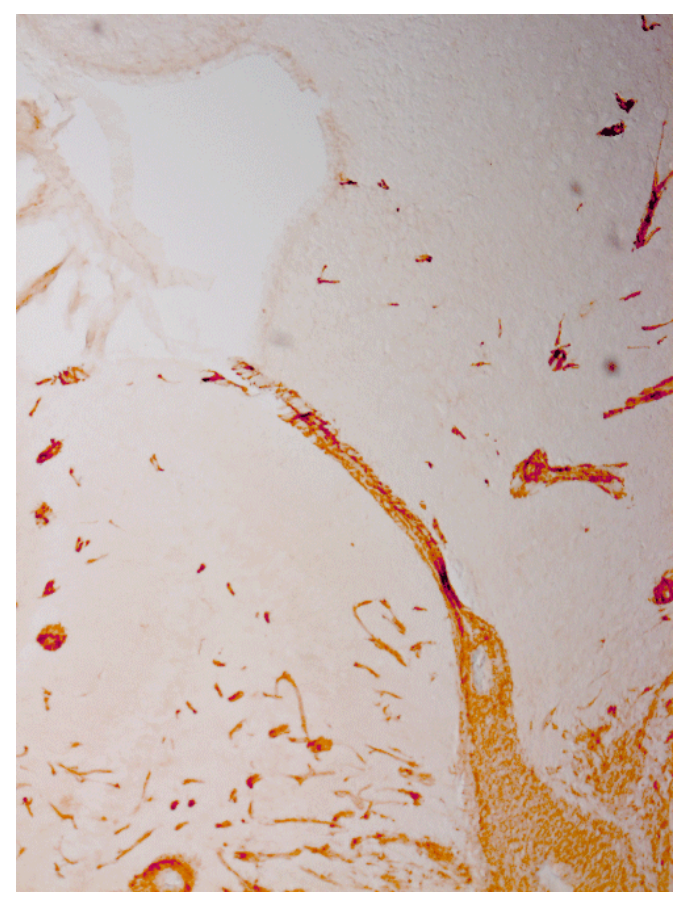

Figure 5: Anti-GFP staining of intracerebrally inplanted NS1 tumour cells.

The tumour cells were transplanted to white GFP negative Fischer 344 recipients, either intracranially (i.c.) and subcutaneously (s.c.). Only those cell lines able to re-generate tumours upon transplantation were considered. This lead to 1 cell line, which grew both intracerebrally and subcutaneously, which was designated as the NS1 (Nittby-Salford 1), with origination in pons. The intracranial tumour was analysed as described below, and the subcutaneous tumour was passed three times in animals, which means it was cultured and injected subcutaneously again in three different animals, upon which it was transplanted intracranially again and generated intracranial tumours as described below (Graph 1). When put under fluorescent microscope, the cells were fluorescent.

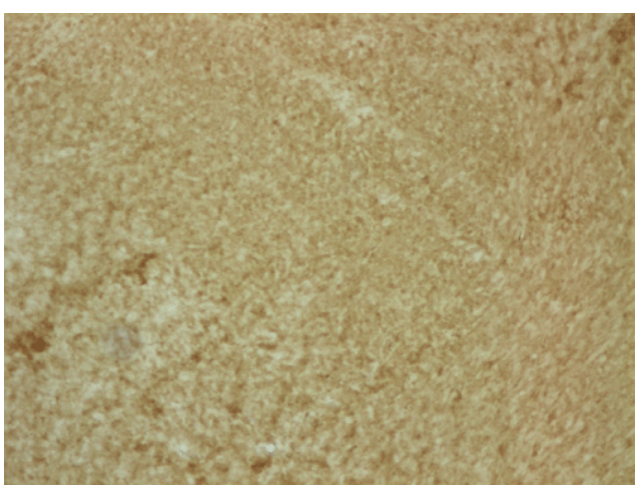

Figure 6: Anti-GFP staining of sc inplanted NS1 tumour cells.

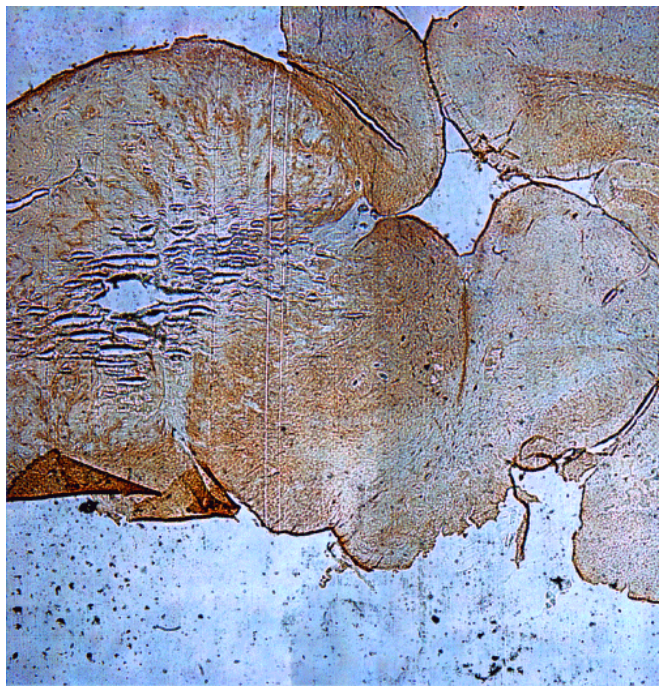

Figure 7: Anti-GFAP staining of intracerebrally inplanted NS1 tumour cells.

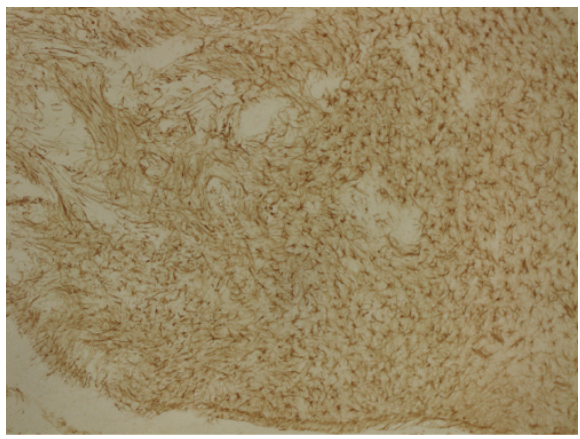

Figure 8: Anti-GFAP staining of intracerebrally inplanted NS1 tumour cells. 
Citation: Nittby H, Fornvik K, Ahlstedt J, Ceberg C, Ericsson P, et al. (2015) A GFP Positive Glioblastoma Cell Line NS1 - A New Tool for

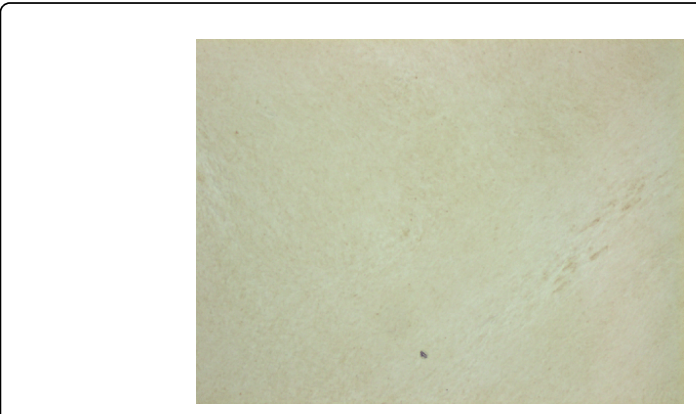

Figure 9: Anti-GFAP staining of subcutaneously inplanted NS1 tumour cells.

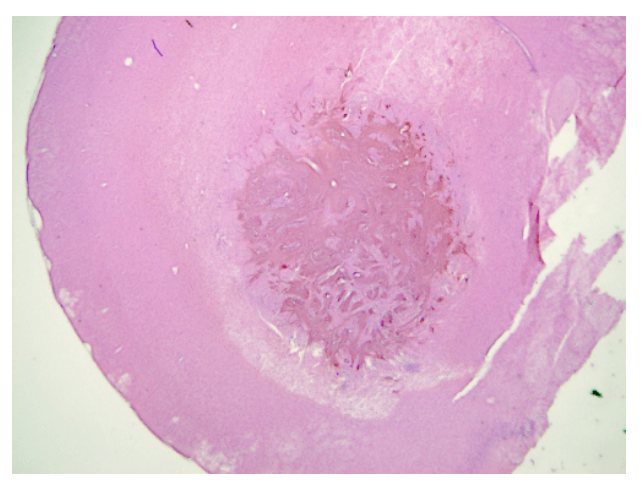

Figure 10: Staining with both GFP antibodies and htx-eosin clearly shows the infiltrating GFP postivie tumour cells (NS1 after three passages in animals, rat sacrificed at day 14 after tumour cell inoculation without any clinical signs of tumour growth).

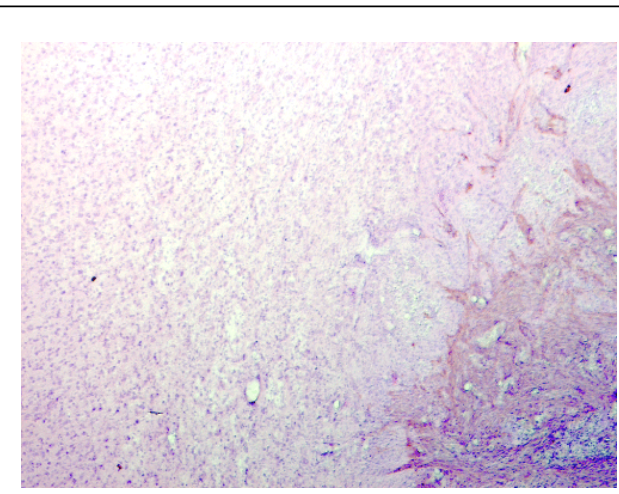

Figure 11: Staining with both GFP antibodies and htx-eosin clearly shows the infiltrating GFP postivie tumour cells (NS1 after three passages in animals, rat sacrificed at day 14 after tumour cell inoculation without any clinical signs of tumour growth).

With htx-eosin staining cell rich tumours could be identified both i.c. and s.c. (Figures 1-3), with a central necrosis and pseudopallisading of the cells, both in cells cultured directly from the tumour and cells passed three times subcutaneously in GFP negative rats.

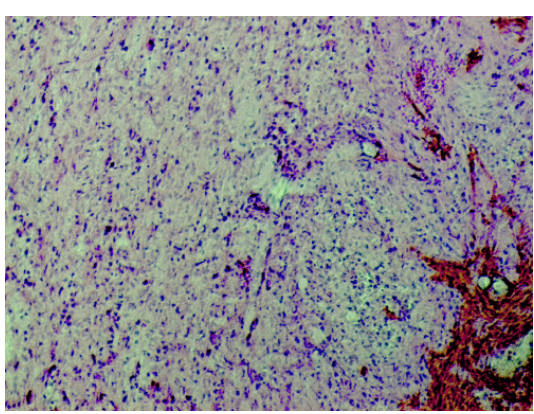

Figure 12: Staining with both GFP antibodies and htx-eosin clearly shows the infiltrating GFP postivie tumour cells (NS1 after three passages in animals, rat sacrificed at day 14 after tumour cell inoculation without any clinical signs of tumour growth).

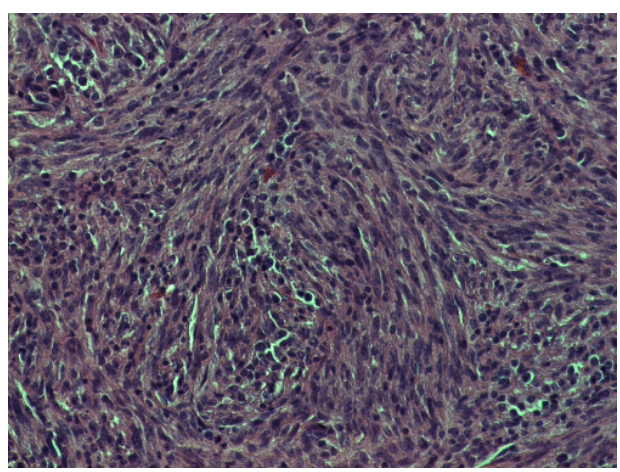

Figure 13: Stating with htx-and eosin demonstrating densely packed tumour cells in this paraffin section of $5 \mu \mathrm{m}$, already after 1 week $(5 \mathrm{~A})$.

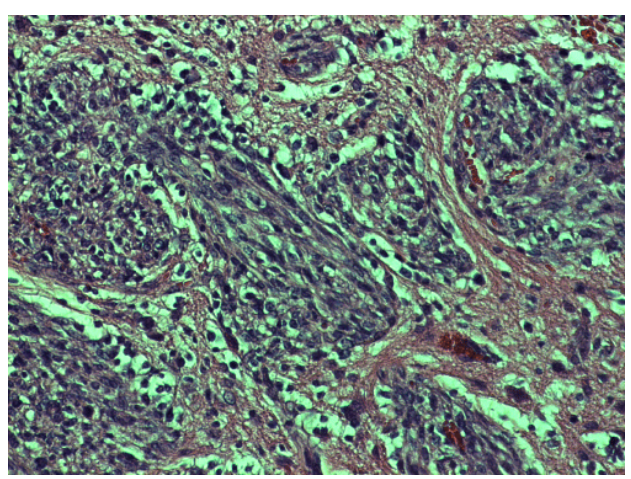

Figure 14: Stating with htx-and eosin demonstrating densely packed tumour cells in this paraffin section of $5 \mu \mathrm{m}$, already after 2 week $(5 \mathrm{~B})$

With GFP staining of intracerebrally transplanted tumour cells into a GFP negative Fischer 344 rat, a GFP positive tumour bulk could be identified, and in the front zone of the tumour, infiltrating GFP positive cells could be identified (Figures 4 and 5). This was constant also in cells which had passed three GFP negative animals before being 
Page 6 of 8

injected intracranially. Also tumours transplanted subcutaneously into white Fischer 344 rats expressed GFP (Figure 6). With GFAP staining, we found positive stainings for the intacerebrally transplanted tumour cells (Figures 7 and 8), also after subcutaneous passing of the cell line in three GFP negative animals. Interestingly, this was less evident in the subcutaneously transplanted cells (Figure 9). With GFP and htxeosin double staining, the infiltrative growth of the GFP positive tumour cells could clearly be seen (Figures 10-12).

Additionally, we could demonstrate tumour cells already 1 and 2 weeks after inoculation with the GFP cells (Figures 13 and 14).

With FACS analysis we could detect GFP expression, as expected, in the GFP positive cells. Figure 6 shows FL1 (detects GFP) against FL4. Cells that lie to the left are GFP negative (see RG2 cells) and cells that lie to the right are GFP positive. Some GFP cells are intermediately GFP positive and most are intensely positive. There are some cells that do not express GFP in the GFP sample (to the left) and some cells in the RG2 sample that seem to express GFP. One possible explanation could be contaminating cells (Figure 15).
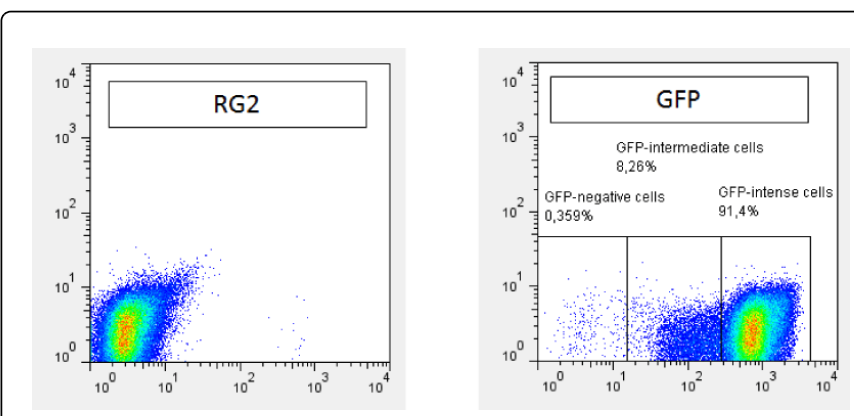

Figure 15: FACS analyses of RG2 cells (left) and GFP cells (right) showing intense expression of GFP in most GFP positive cells, and almost no GFP expression at all in the RG2 cells.

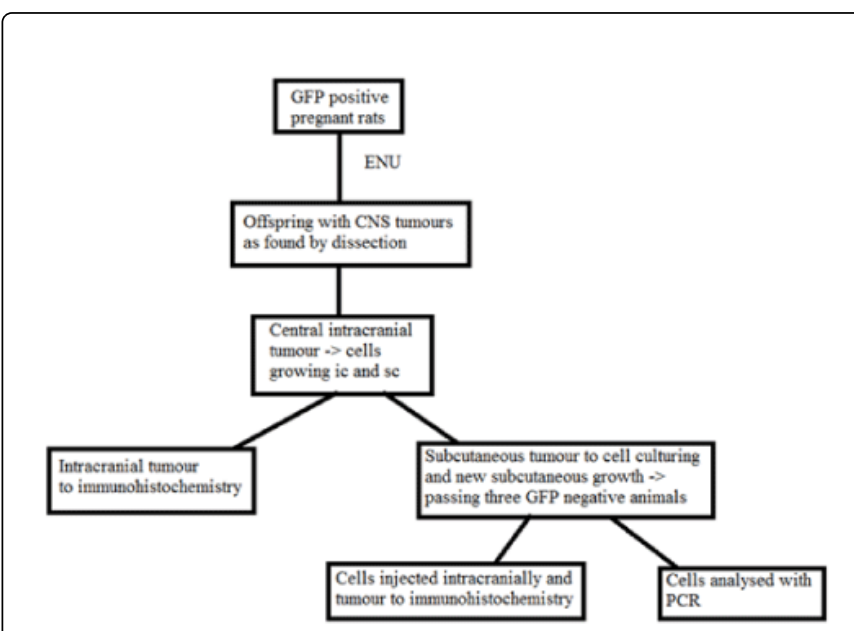

Graph 1: Schematic table of the results.

With PCR analysis, RNA of IDO1 could be identified in the NS1 cell line, and a decrease could be seen after 8 Gy of irradiation (Graph 2) (analysis of the final cell line passed three times subcutaneously). PDL1 were identified and the expression was further induced by irradiation of the cells with $8 \mathrm{~Gy}$. Also, expression of EGFR could be identified and this expression was slightly decreased after 8 Gy of irradiation. Moreover, wt IDH1and wild type un-mutated p53 were identified in relatively high levels, and IDH1 was slightly up-regulated and p53 was slightly down-regulated after irradiation. This is to be compared to the RG2 cell line, where also high levels of wt IDH1and IDO1 were identified, and PDL1 could be seen at lower levels as compared to IDH1 and IDO1. However, the RG2 cells had a much lower expression of EGFR, and irradiation with 8 Gy lead to a slight increase. The decrease in IDO1 expression after irradiaton was much more moderate in the RG2 cells as compared to the effect seen in the NS1 cell line.

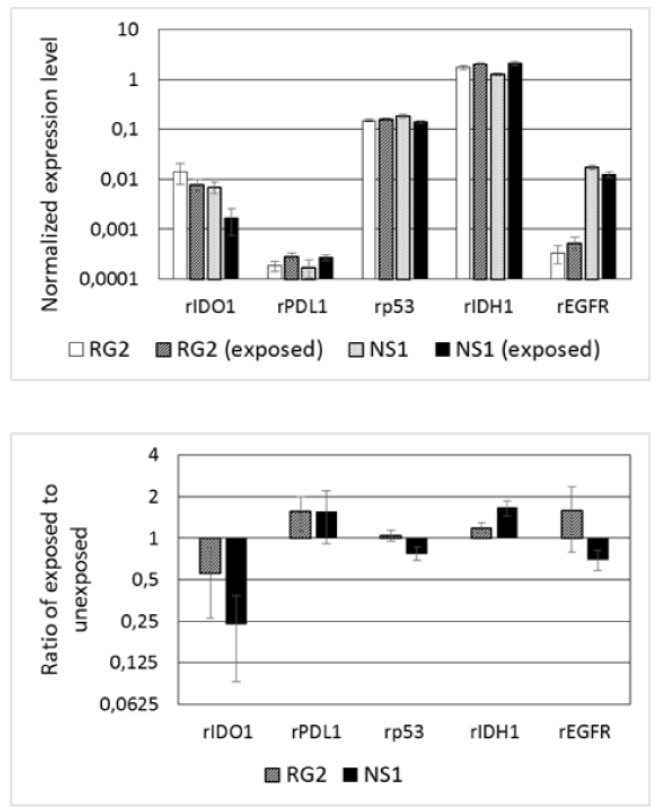

Graph 2: Normalized expression vs HPRT for RG2 cells with or without $8 \mathrm{~Gy}$ of irradiation and green NS1 cells with or without $8 \mathrm{~Gy}$ of irradiation on a logarithmic scale.

Seven animals were further followed after implantation of 300000 ic into the right nucleus caudatus. They all developed signs of tumour growth with hemiparesis and a decline in general condition within 19-23 days from tumour cell inoculation, demanding euthanasia according to our ethical approval (mean survival 21 days, SD 1.8 days).

\section{Discussion}

With a new GFP positive tumour cell line we have created a tool for future investigations of the migrating tumour cells in experimental model. With this new tool, our hope is that treatment protocols will more easily evaluate not only the effect on survival but also whether satellite cells are targeted or not. A major advantage with the NS1 model is that it is used in immunocompetent animals, meaning that it is an excellent tool for studies of immunotherapy against gliomas.

Providing a suitable and strongly expressed immunohistochemical target for staining makes it possible not only to study glioma progression and spread on the single cell level, but also interaction with host tissue. The method with which the GFP gene has been introduced in this line requires no selection of the cell population with regard to GFP expression, unlike previously described models using transfection of genes into already existing tumour populations. Minimizing 
selection of tumour cells allows us to maintain heterogenicity of the tumour population with intact GFP expression.

It has been described that in human GBM, the degree of GFAP reactivity is highly variable. Astrocyte-like tumour cells are generally GFAP positive, while small undifferentiated cells tend to be negative or stain only weakly. Even though large portions of GBM may lack GFAP positive cells, at least some occasional cells are positive. Furthermore, GFAP expression often decreases during glioma progression. Ki67/ MIB-1 shows great regional variation, but mean values of $15-20 \%$ were reported [12]. An interesting finding was that when we transplanted the NS1 GFP cell line intracerebrally in Fischer 344 rats, the tumours showed GFAP positivity. However, upon subcutaneous transplantation, the GFAP staining was much less evident. This is in concordance with the findings by Wechsler et al., who found that RG2 cells were not positive for GFAP in monolayer cultures, but when they were transplanted intracerebrally, GFAP was positive both in a reactive non-neoplastic way but also in clearly neoplastic cells [5].

One aspect that differentiates the NS1 line from the RG2 line is the relatively high expression of EGFR. We mean that this would imply that the NS1 cell line is a better model to study gliomas as compared to the RG2 cell line, since it has been shown that $40 \%$ of human gliomas show amplification of the EGFR gene, and express EGFR even at levels high enough to justify clinical studies with EGFR inhibitors $[6,13]$. The overexpression of EGFR is characteristic of primary glioblastoma, and not secondary, which is another aspect that the NS1 glioma model has in common with its human counterpart.

As shown above, PDL-1 expression is seen in the NS1 tumour cell line, and slightly increased after a single dose irradiation. This is in line with the RG-2 model. Also in models of human gliomas, PDL1 has been identified [10]. PDL-1 has been shown to play a role in suppressing the host immune reaction in gliomas, and is a promising target in immunological treatment research.

Oghaki et al. [7] described that IDH1 mutation was correlated to low-grade astrocytomas, and seen in secondary high-grade astrocytomas in as many as $80 \%$ of the cases, whereas it was rarely seen in primary high grade astrocytomas $(<5 \%)$. Schumacher et al. [14] describe IDH1 as a potential target for vaccinations, and refer to that more than $70 \%$ of diffuse grade II and grade III gliomas carry a mutated form of IDH1. From this aspect, our NS1 line responds well to the criteria of a primary high grade astrocytoma. Furthermore, TP53 mutations were described to be uncommon in primary glioblastomas, which is in line with our NS1 cell line where the levels of unmutated p53 were high. Mutated p53, on the other hand, occurred with a high incidence in secondary glioblastomas. Also, EGFR receptor overexpression was seen in primary glioblastomas, but not in secondary, which is also in line with our NS1 tumour, but makes the RG2 a less favorable model from this aspect [7].

From a treatment point of view, it is interesting that IDO1 was expressed both in the RG2 cells and the NS1 cells, since many IDO1inhibitors are available, one of them being 1-MT, which is already in clinical use in cases of malignant melanomas, which also express IDO1. In the NS1 cell line, IDO1 was actually down-regulated by irradiation. This suggests that irradiation in conjunction with adjuvant targeted immunotherapy may have clinical use in treatment.

Radiation exposure induces DNA damage as well as effects on numerous cellular signaling pathways [15]. Among the radiationinduced pro-survival signaling pathways, some are involved in inducing cell cycle arrest and promoting DNA repair, while others are engaged in suppressing apoptosis induction [16-17]. These pathways act synergistically to protect cancer cells from the cytotoxic effects of radiation, ultimately leading to the development of radio-resistance [18]. Radiation-induced signaling is associated with the transcriptional up-regulation of genes involved in DNA repair such as Ataxiatelangiectasia mutated (ATM) that plays a key role in regulating the cellular response to ionizing radiation [19].

To our knowledge, a GFP expressing glioma cell line has not been described previously in immunocompetent animals, and not in a rat model. However, with immunedeficient mice, it has been seen that within the tumour, not only the initial tumour cells are seen, but also it seems that the host cells are recruited. Cehn et al. [20] described how mouse glioma cells were transfected with RFP and then transplanted into athymic nude mice with GFP expression. They concluded that transplanted tumor tissues contained not only transplanted glioma stem cells but also cancerous host GFP cells. Dong et al. [21] transplanted human glioma cells into GFP transgenic nude mice, and concluded that a cell fusion occurred between hGSCs and host cells. However, as compared to our model, the mouse host animals were not immunocompetent, meaning that the study of immunology in this model is very difficult to interpret.

With this report, we wish to extend the arsenal of tools used in the search of a treatment for this aggressive malignancy. We present a natively GFP positive tumour cell line, NS1. This model shows potential as it offers new ways of evaluating treatments on a microscopic level in vivo. The biochemical profile as studied in this report also suggests that it may in some ways be more predictive of human glioblastoma than other available models, and is a suitable tool for evaluating immunological treatments for glioblastoma.

\section{Conclusion}

With a new GFP positive tumour cell line, NS1, we have a new tool for investigations of the migrating tumour cells in experimental models of primary high grade astrocytomas. With this new tool, our hope is that treatment protocols will more easily evaluate not only the effect of survival (which is often a limited number of days in animal models) but also whether satellite cells are targeted or not in immunocompetent animals.

\section{Acknowledgements}

We are grateful to the Hans and Märit Rausing Charitable Trust, the Mrs Berta Kamprad Foundation, Janne Troedssons forskningsfond and the Crawfoord foundation for financial support. We are grateful to Camilla Björklöv and Agnieszka Czopek for excellent handling of the animals.

\section{References}

1. Stupp R, Mason WP, van den Bent MJ, Weller M, Fisher B, et al. (2005) Radiotherapy plus concomitant and adjuvant temozolomide for glioblastoma. N Engl J Med 352: 987-996.

2. Barth RF, Kaur B (2009) Rat brain tumor models in experimental neurooncology: the C6, 9L, T9, RG2, F98, BT4C, RT-2 and CNS-1 gliomas. J Neurooncol 94: 299-312.

3. Verreault M, Strutt D, Masin D, Fink D, Gill R, et al. (2011) Development of glioblastoma cell lines expressing red fluorescence for non-invasive live imaging of intracranial tumors. Anticancer Res 31: 2161-2171. 
Citation: Nittby H, Fornvik K, Ahlstedt J, Ceberg C, Ericsson P, et al. (2015) A GFP Positive Glioblastoma Cell Line NS1 - A New Tool for Experimental Studies. Brain Tumors Neurooncol 1: 101.

Page 8 of 8

4. Bryant MJ, Chuah TL, Luff J, Lavin MF, Walker DG (2008) A novel rat model for glioblastoma multiforme using a bioluminescent F98 cell line. J Clin Neurosci 15: 545-551.

5. Wechsler W, Kleihues P, Matsumoto S, Zülch KJ, Ivankovic S, et al. (1969) Pathology of experimental neurogenic tumors chemically induced during prenatal a postnatal life. Ann NY Acad Sci 159: 360-408.

6. Sampson JH, Heimberger AB, Archer GE, Aldape KD, Friedman AH, et al. (2010) Immunologic escape after prolonged progression-free survival with epidermal growth factor receptor variant III peptide vaccination in patients with newly diagnosed glioblastoma. J Clin Oncol 28: 4722-4729.

7. Ohgaki H, Kleihues P (2011) Genetic profile of astrocytic and oligodendroglial gliomas. Brain Tumor Pathol 28: 177-183.

8. Avril T, Saikali S, Vauleon E, Jary A, Hamlat A, et al. (2010) Distinct effects of human glioblastoma immunoregulatory molecules programmed cell death ligand-1 (PDL-1) and indoleamine 2,3-dioxygenase (IDO) on tumour-specific T cell functions. J Neuroimmunol 225: 22-33.

9. Zeng J, See AP, Phallen J, Jackson CM, Belcaid Z, et al. (2013) Anti-PD-1 blockade and stereotactic radiation produce long-term survival in mice with intracranial gliomas. Int J Radiat Oncol Biol Phys 86: 343-349.

10. Berghoff AS, Kiesel B, Widhalm G, Rajky O, Ricken G, et al. (2015) Programmed death ligand 1 expression and tumor-infiltrating lymphocytes in glioblastoma. Neuro Oncol 17: 1064-1075.

11. Aas AT, Brun A, Blennow C, Strömblad S, Salford LG (1995) The RG2 rat glioma model. J Neurooncol 23: 175-183.

12. Kleihues P, Cavenee WK. Pathology and Genetics. Tumours of the Nervous System. Lyon, France. P 23.
13. Humphrey PA, Wong AJ, Vogelstein B, Friedman HS, Werner MH, et al. (1988) Amplification and expression of the epidermal growth factor receptor gene in human glioma xenografts. Cancer Res 48: 2231-2238.

14. Schumacher T, Bunse L, Pusch S2, Sahm F2, Wiestler B3, et al. (2014) A vaccine targeting mutant IDH1 induces antitumour immunity. Nature 512: 324-327.

15. Gewirtz DA1 (2000) Growth arrest and cell death in the breast tumor cell in response to ionizing radiation and chemotherapeutic agents which induce DNA damage. Breast Cancer Res Treat 62: 223-235.

16. Hawkins AJ, Golding SE, Khalil A, Valerie K (2011) DNA double-strand break - induced pro-survival signaling. Radiother Oncol 101: 13-17.

17. Raleigh DR, Haas-Kogan DA (2013) Molecular targets and mechanisms of radiosensitization using DNA damage response pathways. Future Oncol 9: 219-233.

18. Hein AL, Ouellette MM, Yan Y (2014) Radiation-induced signaling pathways that promote cancer cell survival (review). Int J Oncol 45: 1813-1819.

19. Kurz EU, Lees-Miller SP (2004) DNA damage-induced activation of ATM and ATM-dependent signaling pathways. DNA Repair (Amst) 3: 889-900.

20. Chen YM, Fei XF, Wang AD, Dai XL, Zhang JS, et al. (2013) [Host glial cell canceration induced by glioma stem cells in GFP/RFP dual fluorescence orthotopic glioma models in nude mice]. Zhonghua Zhong Liu Za Zhi 35: 5-10.

21. Dong J, Zhang Q, Huang Q, Chen H, Shen Y, et al. (2010) Glioma stem cells involved in tumor tissue remodeling in a xenograft model. J Neurosurg 113: 249-260. 